\title{
Médiévales
}

Langues, Textes, Histoire

63 | automne 2012

Philosophies morales

\section{Habemus sanctam! La vie sans fin de Hildegarde de Bingen}

Habemus sanctam! The Endless Life of Hildegard of Bingen

\section{Laurence Moulinier-Brogi}

\section{OpenEdition}

1 Journals

\section{Édition électronique}

URL : https://journals.openedition.org/medievales/6878

DOI : $10.4000 /$ medievales. 6878

ISSN : 1777-5892

Éditeur

Presses universitaires de Vincennes

\section{Édition imprimée}

Date de publication : 15 décembre 2012

Pagination : 145-154

ISBN : 978-2-84292-353-2

ISSN : 0751-2708

\section{Référence électronique}

Laurence Moulinier-Brogi, « Habemus sanctam! La vie sans fin de Hildegarde de Bingen », Médiévales [En ligne], 63 | automne 2012, mis en ligne le 15 janvier 2013, consulté le 22 avril 2022. URL : http:// journals.openedition.org/medievales/6878; DOI : https://doi.org/10.4000/medievales.6878 
Médiévales 63, automne 2012, p. 145-154

\section{Laurence MOULINIER-BROGI}

\section{HABEMUS SANCTAM! \\ LA VIE SANS FIN DE HILDEGARDE DE BINGEN}

Connue de longue date pour ses visions, Hildegarde de Bingen (10981179) a conquis ces dernières années un public de plus en plus large grâce à deux autres de ses dons. Cette bénédictine rhénane, dont la longue vie fut particulièrement active et productive, s'essaya en effet avec autant de succès à la musique et à la médecine, domaines dans lesquels très peu de femmes ont laissé leur nom au Moyen Âge. Ses chants liturgiques (dont elle composa ellemême le texte et la musique) ont été conservés dans des manuscrits contemporains de leur auteur, et sont abondamment joués et enregistrés : ils ont valu au groupe Sequentia un disque d'or en 1996 ${ }^{1}$. Quant à sa médecine, elle a séduit un nombre toujours croissant d'adeptes en quête d'alternatives à la médecine traditionnelle, d'abord dans les pays germaniques puis de proche en proche dans d'autres ${ }^{2}$.

DVD, régimes de santé et sachets d'infusion sont donc les principaux avatars de Hildegarde dans la société occidentale actuelle. Mais si elle est en quelque sorte tombée dans le domaine public par ces deux biais, elle n'en a pas pour autant perdu son attrait pour le monde intellectuel, et la bibliographie la concernant est désormais immense. Dès 1970, Werner Lauter avait eu l'idée de la rassembler, et après un premier tome, il en fit paraître un second en 1982, l'intérêt des chercheurs pour la nonne ayant été aiguillonné par le

1. Canticles of Ecstasy, diffusé en France sous le titre Chants de l'extase, Deutsche Harmonia Mundi, 1994. Conduit par Benjamin Bagby, le groupe Sequentia a produit de très nombreux enregistrements de la musique de Hildegarde, qui est entrée peu à peu dans le répertoire de plusieurs ensembles.

2. Tout récemment est paru en Pologne Św. Hildegarda z Bingen : praktyczny poradnik zdrowego życia, par Brigitte Pregenzer, Brigitte Schmidle ; przekł. Jacek Jurczyński (Kraków, Wydawnictwo M, 2008), titre dont la traduction est « Guide pratique d'une vie saine ». Je remercie Tania Press pour cette précision. 
$800^{\mathrm{e}}$ anniversaire de sa mort célébré en $1979^{3}$. En 1981, le même homme organisa une exposition sur la mémoire de Hildegarde, non dans les livres mais dans les images, et en tira une publication ${ }^{4}$.

Les titres publiés après 1984 furent pour leur part rassemblés par Gertrud Jaron Lewis dans sa Bibliographie zur deutschen Frauenmystik des Mittelalters ${ }^{5}$ et, en 1998, Werner Lauter fit paraître le précieux Hildegard von Bingen. Internationale wissenschaftliche Bibliographie. C'est l'année où fut célébré avec faste le $900^{\mathrm{e}}$ anniversaire de la naissance de la religieuse, année jubilaire que le cloître, qui perpétue la mémoire de la nonne à RüdesheimEibingen, avait ouverte en 1997 avec un volume de mélanges offerts à leur patronne, dans lequel Werner Lauter avait donné une intéressante publication sur l'écho rencontré par Hildegarde en Extrême-Orient ${ }^{6}$. Puis, une fois passées les célébrations de 1998, il tira un petit bilan de cette année mémorable dont les conclusions restent d'actualité?.

Le $900^{\mathrm{e}}$ anniversaire de sa naissance, célébré avec éclat, marqua donc un pic prévisible mais ne donna pas pour autant le la d'un requiem pour une nonne : les publications hildegardiennes continuent d'aller croissant depuis cette date, et l'on peut parler sans se payer de mots d'une mondialisation du phénomène, même si des régions du monde sont plus embrasées que d'autres, souvent sous l'effet du développement des gender studies. Mais notre propos n'est ni historiographique ni bibliographique : ce que nous voulons mettre ici en évidence, c'est d'une part qu'une même figure historique est de nos jours au cœur d'intérêts très divers, mais non contradictoires, et d'autre part qu'elle semble avoir trouvé très récemment deux nouvelles formes de vie post mortem, à l'écran et en cour de Rome. C'est donc sur l'actualité la plus récente d'une figure disparue depuis de nombreux siècles que nous voulons mettre l'accent.

\section{Du texte à l'image}

Ne revenons pas sur l'entrée de Hildegarde dans l'économie de marché et sur tous les «produits dérivés » auxquels son nom sert de label. Soulignons plutôt que cette récupération consumériste n'empêche pas la recherche érudite à son sujet de rester à la fois très vivace et très productive, notamment sous la

3. Hildegard-Bibliographie: Wegweiser zur Hildegard-Literatur und Hildegard-Bibliographie, vol. 2 : 1970-1982, Alzey, 1970-1984. Hildegard von Bingen 1179-1979. Festschrift zum 800. Todestag der heiligen, éd. A. Ph. Brück, Mayence, 1979.

4. W. LAUTER, Das Nachleben der heiligen Hildegard von Bingen. Reproduktionen aus der Rüdesheimer Fotoausstellung, Bingen, 1981.

5. G. J. LewIS, Bibliographie zur deutschen Frauenmystik des Mittelalters (Bibliographien zur deutschen Literatur des Mittelalters), 10, Berlin, 1989, p. 66-145.

6. W. LAUTER, « Schritte der Vermittlung : zur Rezeption Hildegards von Bingen in Japan », dans Hildegard von Bingen. Prophetin durch die Zeiten. Zum 900. Geburtstag, éd. E. ForsTer, Fribourg/Bâle/Vienne, 1997, p. 485-495.

7. ID., « Rückblick auf das Hildegard-Jubiläumsjahr 1997/1998 : Flüchtiges und Bleibendes », Binger Geschichtsblätter, 21 (2000), p. 5-18. 
forme de travaux d'édition de textes. Dans une impressionnante thèse publiée en 2003, Michael Embach a repris à nouveaux frais la question de la tradition manuscrite de l'ensemble des écrits de Hildegarde ${ }^{8}$; et à la toute fin de l'année 2007 est paru dans la célèbre collection « Corpus christianorum continuatio medievalis » des éditions Brepols un volume collectif intitulé Hildegardis Bingensis Opera minora ${ }^{9}$, qui est venu s'inscrire dans la série des écrits de Hildegarde inaugurée il y a trente ans avec la publication de son Scivias par deux sœurs de l'abbaye portant aujourd'hui son nom à Eibingen ${ }^{10}$. Les Opera minora rassemblent cinq écrits dont deux avaient été déjà publiés ensemble par deux fois ${ }^{11}$, mais ce volume manque malheureusement d'une introduction générale qui expliquerait l'esprit de l'entreprise ; on peut donc se demander si ce tome d' ' œuvres mineures » aura ou non une suite regroupant d'autres brefs écrits laissés pour l'heure à l'écart, comme la Vie de saint Disibod ou la Vie de saint Rupert, deux récits hagiographiques consacrés aux saints patrons des deux monastères où elle vécut successivement ${ }^{12}$. On comprend en tout cas implicitement que, si ce volume voit le jour, c'est que leurs éditeurs considèrent que les «grands textes» de Hildegarde sont désormais accessibles et que les écrits ici réunis sont d'une autre envergure.

Ce volume d'Opera minora entérine donc une hiérarchisation des œuvres de la moniale en retrouvant celle du Riesenkodex, le "manuscrit géant » (481 folios de $46 \times 30 \mathrm{~cm}$ ) copié du vivant de la nonne et aujourd'hui conservé à Wiesbaden ${ }^{13}$, qui rassemblait ses livres dans un ordre mêlant progression chronologique et importance décroissante, comme pour en former un canon. Ce que confirme, a contrario, la ségrégation des œuvres médicales

8. М. Емвасн, Die Schriften Hildegards von Bingen. Studien zu ihrer Überlieferung und Rezeption im Mittelalter und in der Frühen Neuzeit, Berlin, 2003.

9. Hildegardis Bingensis Opera minora, éd. P. Dronke, C. P. Evans, H. Feiss et alii, Turnhout, 2007.

10. Hildegardis Scivias, éd. A. FüHrkötter, A. CARLEvaris, Turnhout, 1978, 2 vol. (CCCM t. 43 et $43 \mathrm{~A})$.

11. De Regula sancti Benedicti, et Explanatio symboli sancti Athanasii, publiés ensemble d'abord dans la Maxima Bibliotheca Patrum puis dans la Patrologie latine. Voir d'une part Explicatio Regulae, dans Bibliotheca magna veterum Patrum et scriptorum ecclesiasticorum, post Margerin de la Bigne per theologos Colon., t. XII, Cologne, 1619-1622, rééd. t. XXIII, Lyon, Apud Anissonios, 1677, p. 590-594 ; Explicatio Symboli, ibid., p. 594-600 ; voir d'autre part Explanatio symboli sancti Athanasii, éd. J.-P. Migne, dans Sanctae Hildegardis abbatissae opera omnia, Patrologia latina, t. 197, Paris, 1855 [dorénavant PL 197], col. 1065B-1067A, et Regula S. benedicti juxta S. Hildegardem explicata, ibid., col. 1055-1066.

12. En allemand, le Disibodenberg (Mont-Saint-Disibod) et le Rupertsberg (Mont-Saint-Rupert) ; voir Vita sancti Disibodi, PL 197, col. 1093-1116, et Vita sancti Ruperti, ibid., col. 1083-1092.

13. Aujourd'hui Wiesbaden, Hessische Landesbibliothek, ms. 2 ; ce manuscrit de quelque quinze kilos fut copié à la toute fin de la vie de Hildegarde, entre 1177 ou 1179 et 1180 . Pour une description détaillée, voir Hildegardis Bingensis, Liber divinorum operum, éd. A. Derolez, P. Dronke, Turnhout, 1996, p. XCVII-CI. 
de Hildegarde, qui se sont avérées dans la suite des siècles les seuls de ses écrits véritablement sujets à caution. Sans revenir sur leur histoire, rappelons simplement que les préceptes médicaux de Hildegarde, que le public redécouvre aujourd'hui avec enthousiasme, posent maint problème. Non seulement ils nous ont été transmis par des manuscrits tardifs, donc susceptibles d'avoir subi nombre de modifications, mais surtout, aveu de l'auteur et inventaire après décès ne concordent pas à leur sujet. Pour le dire vite, si Hildegarde elle-même affirmait avoir fait œuvre de science naturelle (« et ce fut la première année après que la même vision m'eut montré, pour que je les explique, les subtilités des diverses natures des créatures », écrit-elle vers $1158^{14}$ ), cinquante ans à peine après sa mort, le livre naturaliste qu'elle revendiquait s'est mué en un diptyque dont les catalogues de bibliothèque médiévaux confirment çà et là l'existence : en lieu et place du Liber subtilitatum reconnu comme sien par la nonne circulent désormais un Liber simplicis medicinae et un Liber compositae medicinae, alias Cause et cure ${ }^{15}$. Ce tandem subit une nouvelle métamorphose à l'époque moderne : entre le $\mathrm{XVI}^{\mathrm{e}}$ et le $\mathrm{XIX}^{\mathrm{e}}$ siècle en effet, non seulement on perd la trace de l'œuvre à deux volets, mais en outre, le traité mis sous le nom de Hildegarde qui sort des presses de l'imprimeur strasbourgeois Jean Schott en 1533 ne correspond à aucun témoin manuscrit médiéval, ni par son titre de Physica ni par son organisation ${ }^{16}$. Enfin, ces ouvrages problématiques n'étaient disponibles encore récemment que dans des éditions fautives ou anciennes, alors que les avancées de la recherche et la découverte de nouveaux manuscrits réclamaient un nouvel établissement des textes : on ne peut donc que saluer la parution, coup sur coup, de deux éditions tant attendues de la Physica ${ }^{17}$.

On n'oubliera pas, enfin, que cette vague d'éditions a touché même un des écrits les plus mystérieux de Hildegarde, à savoir La lingua ignota, la «Langue inconnue », un lexique imaginaire lié lui-même, bien que de manière non univoque, à un alphabet inconnu, et qui subsiste actuellement dans deux manuscrits, l'un à Wiesbaden et l'autre à Berlin ${ }^{18}$. Poursuivant l'œuvre

14. Liber vite meritorum, éd. A. CARLEvaris, Turnhout, 1995, p. 8 : «qui primus annus fuit, postquam eadem uisio subtilitates diversarum naturarum creaturarum, [...] ad explanandum ostenderat ».

15. Voir Beate Hildegardis Cause et cure, éd. L. Moulinier, Berlin, 2003.

16. Voir à ce sujet L. Moulinier, Le Manuscrit perdu à Strasbourg. Enquête sur l'œuvre scientifique de Hildegarde, Paris/Saint-Denis, 1995, notamment chapitre 1, «Les vicissitudes d'une œuvre».

17. Hildegard von Bingen, Physica, Edition der Florentiner Handschrift (Cod. Laur. Ashb. 1323, ca 1300) im Vergleich mit der Textkonstitution der Patrologia Latina (Migne), éd. I. Müller, Chr. Schulze, Hildesheim-Zürich-New York, 2008 ; Ead., Physica, Liber subtilitatum diversarum naturarum creaturarum, éd. R. Hildebrandt, T. Gloning, Textkritische Ausgabe, Berlin-New York, 2 vol., 2010.

18. Respectivement Wiesbaden, Hessische Landesbibliothek, 2, f. 461r-464r (Riesenkodex), et Berlin, Staatsbibliothek Preußischer Kulturbesitz, ms. lat. qu. 674, f. 58r-62r. Sur cette tradition 
d'édition de textes hildegardiens impulsée dans les années 1950 par deux sœurs de l'abbaye Sankt Hildegard ${ }^{19}$, l'alliance des philologues et des historiens a porté ses fruits et nous disposons désormais d'éditions scientifiques même pour les écrits les plus énigmatiques ou les moins connus de Hildegarde.

Plus radicalement neuf est l'accès de Hildegarde à une forme d'immortalité par l'image en mouvement dans le dernier film, sobrement intitulé Vision (2009), que Margarethe von Trotta a tiré de sa vie - un film nominé à la Festa internazionale di Roma en octobre 2009 et déjà vu par nos voisins américains, mais qui n'a curieusement pas été distribué en France, ce pourquoi on nous permettra de le détailler quelque peu.

\section{Vision sur écran}

La grande cinéaste, auteur de l'Honneur perdu de Katharina Blum et des Années de plomb, a trouvé à l'évidence dans la vie de la visionnaire une riche source d'inspiration, et réalisé un film dans lequel Barbara Sukowa prête à merveille ses traits et sa voix à la bénédictine des bords du Rhin. Hannah Herzsprung joue la jeune protégée de Hildegarde, Richardis de Stade, et Heino Ferch fait un Volmar fort convaincant, sans oublier un rôle mineur mais tout aussi fondamental, à savoir celui de Jutta von Spanheim ( $† 1136)$, une recluse qui fut la première magistra de Hildegarde, incarnée par Lena Stolze.

La photographie est de grande qualité et les quelques scènes où la vie de Hildegarde est clairement enjolivée se laissent regarder avec plaisir. Ainsi la scène poignante où Hildegarde doit chasser de son couvent une sœur Clara qui a fauté, celle où elle se présente devant Frédéric Barberousse et n'accepte de s'asseoir que pour jouer aux échecs avec lui après lui avoir «prédit » qu'il serait empereur, ou encore Hildegarde enfant surprenant un moine en train de s'administrer la discipline, signe avant-coureur de son aversion pour les excès d'ascèse auxquels devait succomber sa maîtresse Jutta.

Aussi romancée que superbe est la scène où Hildegarde, en cheveux comme ses sœurs, se livre avec ces dernières et Volmar à un jeu liturgique en l'honneur de Tengswind (Tenxwindis) d'Andernach, qui y assiste sans se départir d'un regard très sévère avant d'admonester Hildegarde en lui rappelant les préceptes pauliniens. On sait de fait que les manières d'agir de Hildegarde ont quelquefois suscité la désapprobation et que, vers 1148-1150, Tenxwindis, magistra d'Andernach, lui écrit car elle avait été choquée d'apprendre que les nonnes du Rupertsberg, le premier monastère fondé par Hildegarde, se paraient

manuscrite, voir M. Емвасн, Die Schriften Hildegards von Bingen..., p. 252 sq., et sur l'œuvre, voir récemment S. L. Higley, Hildegard of Bingen's Unknown Language : An Edition, Translation, and Discussion, New York, 2007.

19. Voir M. Schrader, A. FüHrkötter, Die Echtheit des Schrifttums der heiligen Hildegard von Bingen : Quellenkritische Untersuchungen, Cologne-Graz, 1956. 
de voiles et de diadèmes ${ }^{20}$. Et d'invoquer l'autorité des Pères et des Apôtres, chez qui elle n'avait rien trouvé de te ${ }^{21}$. Se fondant sur cet échange épistolaire historiquement attesté, Margarethe von Trotta en fait une scène d'une infinie grâce, où le spectateur découvre les deux tresses blondes et le visage de la magistra du Rupertsberg, qui rétorque à la vieille abbesse, en jouant avec la tresse de la jeune Richardis, que Dieu aime la beauté et que la laideur n'existait pas au Paradis. Sont, de même, particulièrement émouvantes et réussies les scènes qui opposent Hildegarde à sa fille chérie Richardis, une jeune noble que ses relations familiales promurent très prématurément à l'abbatiat de Bossum. Hildegarde l'aimait «comme Paul aimait Timothée » - l'échange de lettres conservé tant entre les deux femmes qu'entre Hildegarde et le frère de Richardis, Hartwig, archevêque de Brême, le montre assez ${ }^{22}$-, et Barbara Sukowa sait aussi prêter ses traits à l'amour blessé d'une mère spirituelle.

On peut certes regretter, pour finir, que la réalisatrice nous laisse, sinon au milieu du gué, du moins pas au terme de la vie de la nonne. La dernière très belle scène du film montre Hildegarde à cheval, entamant la tournée de prédications qui la mènera dans plusieurs villes d'Allemagne pour lutter contre l'hérésie qui s'y était développée dans les années 1160 . Elle a déjà plus d'une soixantaine d'années, mais on sait qu'elle vécut jusqu'à 81 ans et que d'autres épisodes saillants de son existence auraient pu encore être portés à l'écran.

\section{Voie vers la béatitude}

Manière de suggérer que la vie de Hildegarde est sans fin ? Il semble en tout cas que, tout récemment, une forme supplémentaire de survie se soit offerte à elle en la personne... du pape ! Pour apprécier le phénomène à sa juste mesure, rappelons que si Hildegarde fut autorisée par le pape Eugène III, lors d'un synode réuni à Trèves en 1147-1148, à dire tout ce qu'elle tenait de l'Esprit Saint, sa canonisation n'aboutit jamais, malgré trois tentatives ${ }^{23}$. Or, à plusieurs reprises,

20. Voir Ep. LII, dans Hildegardis Bingensis Epistolarium, éd. L. VAN AcKer, Turnhout, 1991,p. 126 : « aliud etiam quoddam insolitum de consuetudine vestra ad nos pervenit ».

21. Ibid: «Omnia quippe precedentium Patrum instituta, quibus cunctos spiritales maxime informari condecet, pro posse nostro perscrutantes subtilius, nil in eis tale quod reperimus ». On nous permettra de renvoyer à notre traduction dans « Hildegarde de Bingen, Chants et Lettres (choix) », traduit du latin, présenté et annoté, dans Voix de femmes au Moyen Âge. Savoir, mystique, poésie, amour, sorcellerie XII -XV siècle, éd. D. RÉGNIER-BOHLER, Paris, 2006, p. 77-124, p. 119-120: «La vierge ne couvre pas ses cheveux, symbole de sa verdeur, en vertu d'un commandement, mais elle se couvre de son propre chef, en vertu de sa suprême humilité [...] C'est pourquoi il est juste, comme l'a révélé et autorisé le souffle mystique du doigt de Dieu, qu'une vierge porte des habits blancs, symbole éclatant de ses fiançailles avec le Christ [...] ».

22. Voir notamment les lettres XI-XIII, dans Hildegardis Bingensis Epistolarium..., p. 26-31; voir notre traduction dans « Hildegarde de Bingen, Chants et Lettres (choix)», p. 111-115.

23. Grégoire IX ordonna une enquête le 27 janvier 1228, le procès de canonisation s'ouvrit en 1233 mais le rapport fut jugé insuffisant. Bien qu'amendé, il ne parvint apparemment pas à 
l'actuel pape Benoît XVI s'est appuyé sur Hildegarde comme sur un modèle, voire comme une autorité. Le $1^{\text {er }}$ septembre 2010, par exemple, à Castel Gandolfo, il dédia sa catéchèse à la nonne après avoir rappelé la lettre apostolique de son prédécesseur Mulieris dignitatem (1988), qui examinait «le rôle précieux que les femmes avaient joué et continuaient de jouer dans la vie de l'Église $»^{24}$. Au cours des siècles que nous avons coutume d'appeler Moyen Âge, dit Benoît XVI, certaines figures féminines se distinguent par la sainteté de leur vie et la richesse de leur enseignement, et Hildegarde de Bingen était l'une d'elles.

Quelques jours plus tard, le 8 septembre, le pape lui a consacré son audience générale au Vatican, déclarant vouloir approfondir sa réflexion sur Hildegarde et citant tant sa lettre à saint Bernard que des extraits de sa première œuvre visionnaire, Scivias ${ }^{25}$ :

\section{Chers frères et sœurs,}

Je voudrais aujourd'hui reprendre et poursuivre la réflexion sur sainte Hildegarde de Bingen, figure importante de femme au Moyen Âge, qui se distingua par sa sagesse spirituelle et la sainteté de sa vie. Les visions mystiques d'Hildegarde ressemblent à celles des prophètes de l'Ancien Testament: s'exprimant à travers les expressions culturelles et religieuses de son époque, elle interprétait à la lumière de Dieu les Saintes Écritures, les appliquant aux diverses circonstances de la vie. Ainsi, tous ceux qui l'écoutaient se sentaient exhortés à pratiquer un style d'existence chrétienne cohérent et engagé. Dans une lettre à saint Bernard, la mystique de Rhénanie confesse : «La vision envahit tout mon être: je ne vois plus avec les yeux du corps, mais elle m'apparaît dans l'esprit des mystères [...]. Je connais la signification profonde de ce qui est exposé dans le psautier, dans l'Évangile, et d'autres livres, qui m'apparaissent en vision. Celle-ci brûle comme une flamme dans ma poitrine et dans mon âme, et m'enseigne à comprendre en profondeur le texte. »

Les visions mystiques de Hildegarde sont riches de contenus théologiques. Elles font référence aux événements principaux de l'histoire du

Rome dans sa nouvelle version et Innocent IV, dans sa bulle Supplicantibus olim (24.XI.1243), ordonna en vain que l'enquête fût recommencée. Puis, sous le pontificat de Jean XXII, de nouvelles démarches furent faites auprès du Saint-Siège, qui n'aboutirent qu'à la concession d'indulgences collectives envoyées d'Avignon par douze évêques, le 5 décembre 1324. Voir à ce sujet P. BRUDER éd., «Acta Inquisitionis de virtutibus et miraculis sanctae Hildegardis », Analecta Bollandiana, 2 (1883), p. 116-129, p. 129, et A. Vauchez, La Sainteté en Occident aux derniers siècles du Moyen Âge, Rome, 1981, rééd. 1988, p. 108, n. 27. Sur l'échec de ces démarches, on me permettra de renvoyer aussi à L. Moulinier, «Et papa libros eius canonizavit : réflexions sur l'orthodoxie des écrits de Hildegarde de Bingen », dans S. Elm, E. Rebillard, A. Romano éd., Orthodoxie, Christianisme, Histoire. Orthodoxy, Christianity, History, Rome, 2000, p. 177-198.

24. Texte français : http://www.vatican.va/holy_father/benedict_xvi/audiences/2010/documents/ hf_ben-xvi_aud_20100901_fr.html (consulté le 2 juillet 2012).

25. Version française de l'audience générale sur http://www.vatican.va/holy_father/benedict_ xvi/audiences/2010/documents/hf_ben-xvi_aud_20100908_fr.html (consulté le 2 juillet 2012). 
salut, et adoptent un langage principalement poétique et symbolique. Par exemple, dans son œuvre la plus célèbre, intitulée Scivias, c'est-à-dire « Connais les voies », elle résume en trente-cinq visions les événements de l'histoire du salut, de la création du monde à la fin des temps. Avec les traits caractéristiques de la sensibilité féminine, Hildegarde, précisément dans la partie centrale de son œuvre, développe le thème du mariage mystique entre Dieu et l'humanité réalisé dans l'Incarnation. Sur l'arbre de la Croix s'accomplissent les noces du Fils de Dieu avec l'Église, son épouse, emplie de grâce et rendue capable de donner à Dieu de nouveaux fils, dans l'amour de l'Esprit-Saint.

Enfin, le 20 décembre de la même année, dans son adresse à la curie romaine, c'est encore sur elle qu'il s'est appuyé, sur fond d'affaires de pédophilie, pour dénoncer les scandales secouant l'Église actuelle. Et cette fois, c'est dans une lettre écrite par Hildegarde à la fin de sa vie, en 1170, à Werner de Kirchheim et à sa communauté, que Benoît XVI a trouvé les mots pour décrire ce que l'Église romaine avait vécu en 2010 :

Dans ce contexte, m'est venue à l'esprit une vision de sainte Hildegarde de Bingen qui décrit de façon bouleversante ce que nous avons vécu cette année. «En 1170 après la naissance du Christ, j'étais pendant un long temps malade au lit. Alors, physiquement et mentalement éveillée, je vis une femme d'une beauté telle que l'esprit humain n'est pas capable de comprendre. Sa figure se dressait de la terre jusqu'au ciel. Son visage brillait d'une splendeur sublime. Son regard était dirigé vers le ciel. Elle était vêtue d'un vêtement lumineux et resplendissant de soie blanche et d'un manteau garni de pierres précieuses. Aux pieds elle portait des souliers d'onyx. Mais son visage était couvert de poussière, son vêtement était déchiré du côté droit. Le manteau aussi avait perdu sa beauté singulière et ses chaussures étaient souillées sur le dessus. D’une voix haute et plaintive, la femme cria vers le ciel : "Écoute, ô ciel : mon visage est sali ! Afflige-toi, ô terre : mon vêtement est déchiré ! Tremble, ô abîme : mes chaussures sont souillées !"

Et elle poursuivit : "J'étais cachée dans le cœur du Père, jusqu'à ce que le Fils de l'homme, conçu et engendré dans la virginité, répandît son sang. Avec ce sang, comme sa dot, il m'a prise comme son épouse."

Les stigmates de mon époux demeurent frais et ouverts, tant que sont ouvertes les blessures des péchés des hommes. Justement le fait que les blessures du Christ restent ouvertes est la faute des prêtres. Ils déchirent mon vêtement puisqu'ils sont transgresseurs de la Loi, de l'Évangile et de leur devoir sacerdotal. Ils enlèvent la splendeur à mon manteau, parce qu'ils négligent totalement les règles qui leur sont imposées. Ils souillent mes chaussures, parce qu'ils ne marchent pas sur les droits chemins, c'est-à-dire sur les durs et exigeants chemins de la justice, et ils ne donnent pas aussi un bon exemple à ceux qui leur sont soumis. Toutefois je trouve en certains la splendeur de la vérité.

Et j'entendis une voix du ciel qui disait : "Cette image représente l'Église. C'est pourquoi, ô être humain qui vois tout cela et qui écoutes les paroles de plainte, annonce-le aux prêtres qui sont destinés à la conduite et à l'instruction du 
peuple de Dieu et auxquels, comme aux Apôtres, il a été dit: 'Allez dans le monde entier. Proclamez la Bonne Nouvelle à toute la création'” $(\mathrm{Mc} 16,15)^{26}$ ».

Dans la vision de sainte Hildegarde, le visage de l'Église est couvert de poussière, et c'est ainsi que nous l'avons vu. Son vêtement est déchiré - par la faute des prêtres. Ainsi comme elle l'a vu et exprimé, nous l'avons vu cette année. Nous devons accueillir cette humiliation comme une exhortation à la vérité et un appel au renouvellement ${ }^{27}$.

Inutile de multiplier les citations : pour Benoit XVI, Hildegarde est véritablement une référence, y compris par son combat contre les cathares ou ses admonestations au schismatique Barberousse, et il s'avère fin connaisseur de l'ensemble de son œuvre, sur laquelle il jette un regard de théologien averti et dont il fait à son tour une lecture qui lui permet d'y voir une préfiguration des maux actuels. Où l'on renoue avec le thème du tempus muliebre (une expression employée par Hildegarde elle-même dès son Scivias ${ }^{28}$ ), le motif du déclin de l'Église dû à la faiblesse des hommes auquel seules de fortes voix de femmes pourront porter remède, dont les contemporains de Hildegarde commentant sa prise de parole prophétique exprimaient une claire conscience.

On ne saurait prêter au pape une sensibilité féministe proche de celle de Margarethe von Trotta pour expliquer cette réutilisation de Hildegarde en ce début de $\mathrm{XXI}^{\mathrm{e}}$ siècle. Il faut toutefois remarquer qu'après s'être consacré dans deux discours différents à Hildegarde de Bingen, Benoît XVI a évoqué dans sa catéchèse, entre septembre et décembre 2010, plusieurs grandes figures non pas tant de la sainteté officielle que de la mystique médiévale : dans l'ordre, Claire d'Assise (†1253), Mechthilde de Hackeborn (†1299), Gertrude la Grande $(† 1302)$, Angèle de Foligno (†1309), Élisabeth de Hongrie $(† 1231)$, Brigitte de Suède $(† 1373)$, Marguerite d’Oingt (†1310), Julienne de Mont Cornillon (†1258), Catherine de Sienne $(\dagger 1380)$, Julienne de Norwich ( $†$ v. 1416 ?), et le 29 décembre Catherine de Bologne $(† 1463)^{29}$.

26. Lettre à Werner von Kirchheim et à sa communauté, à lire désormais non dans la Patrologie latine mais dans Hildegardis Bingensis Epistolarium..., p. 333-337.

27. Version française du discours du pape : http://www.vatican.va/holy_father/benedict_xvi/ speeches/2010/december/documents/hf_ben-xvi_spe_20101220_curia-auguri_fr.html (consulté le 2 juillet 2012).

28. Voir Scivias, lib. III, cap. 10 et 11.

29. Textes et vidéos des audiences consacrées à ces différentes femmes sur http://www. vatican.va/holy_father/benedict_xvi/audiences/2010/index_fr.htm. Sur ces figures féminines dont l'étude a été puissamment renouvelée ces dernières décennies, il est impossible de renvoyer à une bibliographie débordante, mais on pourra se familiariser avec ces différentes voix en lisant P. DinzelbaCher éd., Frauenmystik im Mittelalter, Stuttgart, 1985 ; A. Vauchez, La Sainteté en Occident... ; C. WALKER BYNum, Jeûnes et festins sacrés : les femmes et la nourriture dans la spiritualité médiévale, trad. fr. Paris, 1994, et D. RÉGNIER-BOHLER, «Voix littéraires, voix mystiques », dans G. Duby, M. Perrot éd., Histoire des femmes en Occident, t. 2, Le Moyen Âge, éd. C. Klapisch-Zuber, Paris, 1991, p. 443-500. 
Il est bien sûr fort possible que Benoît XVI se soit intéressé particulièrement à Hildegarde comme à une «compatriote ». Mais c'est avant tout le caractère visionnaire de ses dires, son emploi de l'allégorie et de l'image, qui rend possible sa réactualisation au $\mathrm{XXI}^{\mathrm{e}}$ siècle, comme en d'autres contextes auparavant, ce dont la nonne s'expliquait elle-même dans une lettre à une autre abbesse : «Et moi je te dis que je n'ai jamais eu l'habitude de m'exprimer en termes nus dans la vision de mon âme, mais avec ceux par lesquels je suis instruite dans cette vision, et toujours aussi selon quelque comparaison, comme il est écrit : «J'ouvrirai la bouche en paraboles, je publierai ce qui fut caché dès l'origine" (Ps. 77 [78], 2). Car Dieu depuis le commencement a proposé aux hommes des paraboles et des comparaisons, par lesquelles le plus grand nombre est instruit vers le salut plus convenablement que par des mots nus ${ }^{30}$. »

En restant vague même dans ses lettres, Hildegarde ne faisait qu'appliquer une règle du message prophétique et, comme l'a souligné Sylvain Gouguenheim, pionnier des études sur la religieuse en France, le goût du langage par paraboles, la dimension oraculaire, voire le flou de ses dires garantirent à ses prophéties une sorte d'atemporalité qui permit la réutilisation de ses écrits dans d'autres contextes et pour servir d'autres besoins ${ }^{31}$.

Elle a ainsi traversé les époques et servi différentes causes, d'aucuns lui faisant prédire a posteriori l'arrivée des mendiants et d'autres, au contraire, l'utilisant au service de la Réforme. De nos jours en tout cas, incontestablement, des marchands de relax bio à la plus haute autorité de l'Église, on lui trouve une actualité et une polyvalence qui en font une figure de recours dans la recherche, non du bien-être, mais du mieux-être spirituel et physique, dans un Occident à qui une certaine opulence n'a pas garanti la santé.

Hildegarde nonne à tout faire, ou guide des égarés du $\mathrm{xxI}^{\mathrm{e}}$ siècle ? L'auteur de ces lignes ne pourra raisonnablement pas voir les célébrations du millième centenaire de la naissance de la moniale, mais si Dieu lui prête vie, elle sera témoin de sa canonisation si longtemps différée. Le 29 mai dernier en effet, au terme de la messe de Pentecôte, le pape a annoncé que, le 7 octobre 2012, il la proclamerait, avec saint Jean d'Avila, docteur de l'Église. Du point de vue français, après tout, « tisane » n'est-il pas l'anagramme de « sainte »?

Laurence Moulinier-Brogi - Université Lumière-Lyon 2, CIHAM (UMR 5648), ISH, 14 avenue Berthelot, 69363 Lyon cedex 07

30. Lettre II, éd. P. DronKe, «Problemata hildegardiana», Mittellateinisches Jahrbuch, 16 (1981), p. 97-131, p. 130 : « ego tibi dico quod nunquam soleo in visione anime mee verbis nudis loqui, sed qualibus in ea doceor, et semper etiam aliqua similitudine, sicut scriptum est : "Aperiam in parabolis os meum, loquar propositiones ab initio." Deus siquidem ab initio hominibus proposuit parabolas et similitudines, per quas plerumque conveniencius quam nudis verbis ad salutem instruuntur.»

31. S. Gouguenheim, La Sibylle du Rhin, Hildegarde de Bingen, abbesse et prophétesse rhénane, Paris, 1996, p. 81. 